\title{
Francophonies barbares, N. Hossard (dir.)
}

\section{Elena Pessini}

\section{OpenEdition}

\section{Journals}

\section{Édition électronique}

URL : http://journals.openedition.org/studifrancesi/10074

DOI : 10.4000/studifrancesi. 10074

ISSN : 2427-5856

\section{Éditeur}

Rosenberg \& Sellier

\section{Édition imprimée}

Date de publication : 1 août 2017

Pagination : 407-408

ISSN : 0039-2944

\section{Référence électronique}

Elena Pessini, «Francophonies barbares, N. Hossard (dir.) », Studi Francesi [En ligne], 182 (LXI | II) | 2017, mis en ligne le 01 août 2017, consulté le 06 janvier 2021. URL : http://journals.openedition.org/ studifrancesi/10074; DOI : https://doi.org/10.4000/studifrancesi.10074

Ce document a été généré automatiquement le 6 janvier 2021.

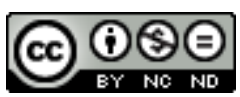

Studi Francesi è distribuita con Licenza Creative Commons Attribuzione - Non commerciale - Non opere derivate 4.0 Internazionale. 


\title{
Francophonies barbares, N. Hossard (dir.)
}

\author{
Elena Pessini
}

\section{RÉFÉRENCE}

Francophonies barbares, sous la direction de Nicolas HOSSARD, «Francofonia» 70, Primavera 2016, Anno XXVI, 207 pp.

1 Comme l'indique Nicolas HOSSARD qui a réuni les sept interventions qui composent ce soixante-dixième numéro de «Francofonia», la démarche qui sous-tend les travaux des différents spécialistes qui se passent la parole répond au besoin de combler une lacune: «la posture et le motif barbares [...] n'ont, dans un champ littéraire "francophone" aux contours récents, jamais fait l'objet d'une étude systématique par la critique spécialisée» (p. 3). Cet essai, intitulé Penser la francophonie par la barbarie?, a le souci à la fois d'introduire les travaux spécifiques présents dans ce numéro, d'illustrer la démarche qui a motivé la recherche mais aussi de creuser une réflexion autour du rapprochement entre le motif barbare et les littératures francophones mais aussi avec la francophonie tout court. Repenser la dialectique francophonie/barbarie est d'autant plus légitime et actuel que cette réflexion se fait au moment où la notion même de francophonie est remise en cause dans l'acception courante qu'elle a eue tout au long du siècle dernier. Ce sont avant tout les idées de centre et de périphérie, d'un centre franco-français autour duquel rayonneraient des satellites plus ou moins éloignés mais tous francophones qui semble ne pas tenir le cap du nouveau millénaire, dévoilant ainsi des implications politiques mais aussi éminemment poétiques.

Maria Chiara GNOCCHI, avec son article Le barbare d'à côté (1900-1939), inaugure les travaux et se concentre sur les années du $\mathrm{xx}^{\mathrm{e}}$ siècle qui précèdent la deuxième guerre mondiale. Les barbares d'à côté qui ont attiré son attention sont les écrivains belges et suisses mais à l'intérieur même du panorama littéraire français, les barbares sont à la fois des écrivains régionalistes et aussi des auteurs aux origines très modestes que rien, à cette 
époque, ne semblait destiner à l'écriture et à la publication. On assiste également, toujours dans ces mêmes années, à une entrée en force des littératures étrangères et des auteurs francophones non français dans le domaine d'intérêt des maisons d'édition parisiennes. L'analyse fouillée de M.C. Gnocchi fait émerger le réseau complexe des relations qui se tissent entre les différents auteurs qui veulent faire entendre leurs voix et dérangent l'ordre statique de la légitimité littéraire. De nombreux points communs unissent ces productions originaires des limites, des marges, en particulier une volonté de subversion, de mise en discussion de la production littéraire existante, un désir de s'en démarquer, mais aussi une occupation prépondérante de la langue écrite par la langue orale. Les conclusions de l'article soulignent fort justement comment des productions littéraires qui sembleraient, apparemment, n'avoir rien en commun si on les examinait avec les instruments de la critique qui avait cours au moment où elles ont été publiées, s'imposent comme sœurs à l'aune d'un regard plus attentif et contemporain. C'est aussi la revendication de leur barbarie qui frappe, les barbares s'affichant comme tels à un moment où le contexte éditorial et de la réception est prêt à les accueillir pour signifier que la barbarie est un des composants de la modernité.

Dans l'analyse de Bernadette CAILLER, Mythes de la barbarie dans "Le Barbare enchanté" de Raphaël Confiant, c'est le roman de l'écrivain martiniquais qui est l'objet d'une étude attentive. Parfaitement centré sur les pistes de recherche que se propose le numéro de la revue, l'article de Cailler s'interroge sur la nature de la barbarie que Gauguin recherche, qui le fascine, qui correspond à une tendance de son siècle, le désir d'aller retrouver le bon sauvage et un Eden à jamais perdu. Raphaël Confiant met en scène le séjour martiniquais du peintre français et laisse au récit fictionnel le soin d'interroger la permanence insulaire de l'artiste pour cerner ce qu'elle a représenté pour l'homme mais surtout pour le créateur qu'il était. Dans son étude, Bernadette Cailler, à travers un travail minutieux qui éclaire la structure narrative du roman et la caractérisation des personnages décrits, souligne que le souci de Confiant est sans doute concentré sur l'approche de la personnalité de Gauguin mais que c'est le regard, regard émerveillé, que ce dernier porte sur ce qui l'entoure, qu'il souhaite illustrer. L'auteure montre que ce roman que Confiant publie en 2003 s'insère parfaitement dans l'ensemble plus vaste que constitue son œuvre en prose qui, au-delà des histoires racontées, poursuit un même but: «Immense est chez Confiant le vœu de participer à la construction d'une littérature nationale martiniquaise qui puisse inclure dans l'imaginaire créatif la diversité humaine et culturelle si riche de son pays, inclure les multiples éléments constituant la vie ou plutôt les vies antillaises d'un temps à l'autre: passé, présent et avenir en gestation» (p. 38).

4 Marjorie JUNG se penche, elle, sur l'œuvre de Frantz Fanon qui, cette étude le prouve, n'a pas encore fini de livrer son message et d'étonner par sa richesse. Dans La figure de l'homme nouveau dans l'œuvre de Frantz Fanon, Jung montre comment les réflexions sur la barbarie alimentent le processus de création et de naissance d'un homme nouveau annoncé et prophétisé par Fanon. La première étape de ce parcours est définie par la mise en place d'une discursivité barbare à laquelle l'auteure consacre une première partie de sa recherche et dont elle fixe les caractéristiques. Dans un second temps, elle s'interroge sur l'émergence, depuis Peau noire, masques blancs jusqu'aux Damnés de la terre, d'une poétique barbare qui s'explicite essentiellement à travers trois étapes: «de l'incorporation à la décorporation pour enfin parvenir à l'excorporation avec la création de la figure de l'homme nouveau» (p. 56). Le questionnement sur la violence clôt cette réflexion, une violence que le colonisé hérite de la colonisation mais qu'il doit 
être capable de convertir: «de la conversion de la violence dépend la création de l'homme nouveau qui seul permet de transcender le mythe du barbare» (p. 63). Avec Chacun est le barbare de l'autre: père et fils dans "L'Africain" de J.M.G. Le Clézio, Valeria SPERTI traque les effets barbares de ce récit bref que Le Clézio a écrit sur commande, le texte ayant paru dans la collection «Traits et Portraits» de Colette Fellous, aux éditions Mercure de France. On peut aisément imaginer que Le Clézio ne se serait sans doute pas livré de sa propre initiative à une plongée, sous bien des aspects douloureuse, dans son passé pour retrouver la figure de son père au moment ou ils ont vécu ensemble en Afrique, au Nigéria, lorsque ce dernier y exerçait sa profession de médecin. Ce bref moment de vie commune de 1948 à 1949, est l'occasion de constater que tout sépare ce père et ce fils. Le Clézio met en scène la rencontre avec une double altérité, l'altérité africaine, terre autre et étrangère, mais aussi l'altérité du père qui ne se prêtera jamais à un véritable échange. Valeria Sperti souligne à travers une démonstration convaincante comment les ressources iconographiques, des photos que le père de Le Clézio a faites à cette époque et qui sont insérées dans l'ouvrage, disent de façon explicite l'écart entre les deux hommes «d'un côté l'écriture assemble, cherche à expliquer, réunissant des évidences, des souvenirs personnels et imaginaires, de l'autre les photos affichent leur caractère décousu et fragmentaire; leur juxtaposition discordante renvoie à l'impossible coïncidence entre le temps et l'espace du père et ceux du fils, à leur étrangeté consubstantielle. Une des significations du livre réside dans cette représentation nostalgique d'un rapport irrécupérable entre deux personnes qui sont restées étrangères l'une à l'autre» (p. 79). Avec la contribution d'Ibrahim DiouF, le lecteur effectue un retour temporel en 1921, année de publication du roman Batouala qui, on le sait, remporta le prix Goncourt. Le titre de cette contribution, Un véritable roman barbare? La langue française à l'écoute de la barbarie dans "Batouala" (1921) de René Maran, explicite les pistes de recherche sur lesquelles Diouf s'est concentré. L'auteur s'est mis à l'écoute des effets de barbarie dans ce que l'écrivain avait défini dans sa célèbre préface «un véritable roman nègre». Ces effets sont à rechercher dans les modalités de la relation entre le colon et le banda pour qui l'autre est foncièrement étranger, incompréhensible, mais également dans la représentation de la nature mise en texte par Maran. «Le roman [...] définit ainsi doublement la barbarie: d'une part, est barbare ce qui est perçu par l'autre comme étranger ou différent; d'autre part, est authentiquement barbare ce qui relève du naturel (le monde vivant, en particulier le monde animal, la sexualité, la voix)» (p. 85). Diouf conduit une analyse stylistique très détaillée de l'écriture de René Maran qui fait de ce roman une œuvre charnière qui annonce, sous certains aspects, la Négritude césairienne, et lui confère son potentiel fondateur.

Chloé VANDENDORPE se penche sur l'œuvre de l'écrivain togolais Kossi Efoui, et c'est particulièrement la langue dans laquelle sont écrits ses romans et ses pièces de théâtre Le Carrefour, Volatiles, La Malaventure, Que la terre vous soit légère, La Fabrique des cérémonies, Solo d'un revenant, que l'auteure s'attache à sonder. Dans Kossi Efoui, une langue barbare à l'usage du monde, elle fait émerger les caractéristiques d'une étrangeté de la langue présente dans les textes qui se révèle dans des pratiques de déréalisation, de détour, de déstabilisation, d'équivoque et d'opacité. L'entretien avec Kossi Efoui que l'on trouve dans la section des interviews et inédits de ce numéro de la revue, et dans lequel l'écrivain se livre avec une très grande générosité au jeu des questions et des réponses, permet au lecteur d'approfondir les problématiques abordées dans l'article critique. Le dossier se termine avec l'intervention de Nicolas HossARD, Genèse d'une 
ambiguité: francophonie et barbarie dans l'œuvre de Jean-Jacques Rousseau, qui aurait sans doute pu figurer en ouverture de cet ensemble de travaux puisque l'écrivain genevois y est présenté comme le tout premier écrivain francophone. Hossard affronte à la fois le thème barbare qu'il définit «immense» chez Rousseau et le thème de la parole pour arriver à des conclusions qui justifient pleinement le parcours tracé: «La francophonie est donc le moyen par lequel la structure discursive barbare s'exerce à l'intérieur de la langue française et, inversement, la barbarie est le vecteur symbolique et discursif par lequel la francophonie émerge en tant qu'état-limite de la littérature de langue française». En conclusion de ces lectures, il nous semble que ce numéro fait plus que combler des lacunes; la variété et la multiplicité des approches et des domaines de recherche ne pourra manquer de susciter un débat qui appelle très certainement des développements ultérieurs. 